\title{
LOCAL RIGIDITY OF SYMMETRIC SPACES OF NONPOSITIVE CURVATURE
}

\author{
VIKTOR SCHROEDER AND MARTIN STRAKE
}

(Communicated by David G. Ebin)

\begin{abstract}
We prove a rigidity theorem for Riemannian manifolds of nonpositive curvature, whose metric is locally symmetric in a neighborhood of the boundary.
\end{abstract}

\section{INTRODUCTION}

The purpose of this paper is to obtain local rigidity results in the sense of [BGS, §5], [SZ] for symmetric spaces of nonpositive curvature. The main result is the following:

Theorem 1. Let $(M, \partial M)$ be a compact Riemannian manifold with sectional curvature $K \leq 0$ (resp. $K \leq-1$ ) and $\operatorname{dim} M \geq 3$. Assume that all principal curvatures of $\partial M$ with respect to the outer unit normal are $\geq 0(\geq-1)$ and that $\partial M$ is compact and simply connected. If the metric of $M$ is locally symmetric of rank $\geq 2$ (rank = 1 , such that the maximum of the sectional curvature is -1) in some neighborhood $U$ of $\partial M$, then $M$ is isometric to a compact domain of a simply connected symmetric space $X$ of rank $\geq 2$ (rank =1) and curvature $K \leq 0 \quad(K \leq-1)$.

This result is a generalization of [SZ, Theorem 5] where the theorem is proved for rank $\geq 3$ and [SZ, Theorem 7] where the rank one case is proved under the assumption that $\partial M$ is convex. In contrast to the local method in [SZ] we use a global argument by embedding $\partial M$ into a symmetric space by the developing map. Our method is even simpler than the proof in [SZ], but it works only for nonpositively curved manifolds and cannot be used to obtain dual rigidity results for symmetric spaces of compact type.

The above theorem implies in particular that $M$ is flat, if the metric is flat in some neighborhood of $\partial M$. If $\operatorname{dim} M=3$, it suffices to assume that the curvature vanishes only on the tangent planes of the boundary. We formulate this in the following way (comp. [BGS], p. 66).

Received by the editors October 1, 1987.

1980 Mathematics Subject Classification (1985 Revision). Primary 53C20, 58G30. 
Theorem 2. Let $S$ be a compact strictly convex immersed surface in a threedimensional Hadamard manifold $X$ (i.e. $X$ is complete, simply connected and of curvature $K \leq 0)$. If the sectional curvature vanishes on all 2-planes tangent to $S$, then $S$ bounds a flat region which is isometric to a convex domain in Euclidean 3-space. In particular $S$ is an embedded sphere.

Let $S$ be any closed convex surface in $X^{3}$ and let $L$ be the Weingarten map of $S$. Then the Gauss-equation and the Gauss-Bonnet formula implies that

$$
I=\int_{S}|\operatorname{det} L| d V \geq 4 \pi
$$

Equality holds if and only if the curvature of all tangent planes vanishes. For an arbitrary convex hypersurface $S$ in an $n$-dimensional Hadamard manifold $X$ one would expect

$$
\int_{S}|\operatorname{det} L| d V \geq \omega_{n-1}
$$

where $\omega_{n-1}$ is the volume of the standard $(n-1)$-sphere. Using ideas from [GW] we can prove this asymptotically by considering parallel hypersurfaces. This gives a partial answer to a question raised in [BGS, p. 66]).

Theorem 3. Let $X^{n}$ be a Hadamard manifold and $S \rightarrow X$ a compact strictly convex immersed hypersurface. Let $S_{t}$ be the outer parallel hypersurfaces of distance $t \geq 0$ with total curvature $I(t)=\int_{S_{t}}\left|\operatorname{det} L_{t}\right| d V_{t}$ where $L_{t}$ denotes the Weingarten map of $S_{t}$. Then $I(t)$ is monotone increasing in $t$. Furthermore $I(\infty)=\lim _{t \rightarrow \infty} I(t) \geq \omega_{n-1}$ and equality holds if and only if $X$ is isometric to $\mathbf{R}^{n}$.

\section{Proof of Theorem 1}

We first recall some facts from the theory of Hadamard manifolds which we will use frequently (compare e.g. [BGS]): A Hadamard manifold is a complete simply connected Riemannian manifold $X$ of nonpositive sectional curvature. The manifold $X$ is diffeomorphic to Euclidean space $\mathbf{R}^{n}$. Any two points $x, y \in X$ can be joined by a geodesic which is unique up to parametrization. A function $f: X \rightarrow \mathbf{R}$ is called convex, if for any geodesic $c: \mathbf{R} \rightarrow X$ the composition $t \mapsto f \circ c(t)$ is convex. A subset $A \subset X$ is convex, if for $x, y \in A$ also the geodesic from $x$ to $y$ is contained in $A$. Examples of convex functions are the distance function $\operatorname{dist}\left(x_{0},\right)$ of any point $x_{0} \in X$ or more generally the distance function $\operatorname{dist}(A$,$) to a convex subset A \subset X$.

We start with a generalization of Hadamars result $[\mathrm{H}]$ (see also [S]) on immersions of convex hypersurfaces to Hadamard manifolds.

Lemma 1. Let $X$ be a Hadamard manifold of dimension $n \geq 3$ and let $\varphi: S \rightarrow$ $X$ be an immersion of a compact hypersurface $S$ which is convex with respect to a unit normal vectorfield along $\varphi$. Then $\varphi$ is an embedding and $\varphi(S)$ is the 
boundary of a compact convex domain in $X$. In particular $S$ is diffeomorphic to a sphere.

Proof. As the immersion $\varphi: S \rightarrow X$ is convex and $X$ has nonpositive curvature, the exponential map

$$
\begin{gathered}
e: S \times[0, \infty) \rightarrow X \\
e(s, t):=e_{t}(s):=\exp _{\varphi(s)} t N(s)
\end{gathered}
$$

is of maximal rank and the parallel hypersurfaces $S_{t}=S \times\{t\}$ are immersed by $e$. We first claim that for $t$ sufficiently large, $e_{t}$ is an embedding. In order to see this choose $x_{0} \in X$, let $\rho(x):=\operatorname{dist}\left(x_{0}, x\right), r_{0}:=\sup \left\{\operatorname{dist}\left(x_{0}, \varphi(s) \mid s \in S\right\}\right.$ and define $t_{0}:=3 r_{0}$. By the triangle inequality $\rho\left(e_{t}(s)\right) \geq \rho(\varphi(s))$ for $t \geq t_{0}$. The convexity of the function $\rho$ (note that $K \leq 0$ ) implies

$$
\left\langle\operatorname{grad} \rho, \frac{d}{d t} e_{t}(s)\right\rangle>0
$$

for all $t \geq t_{0}$. Thus the immersed hypersurface $S_{t}$ is transversal to the vectorfield $\operatorname{grad} \rho$. This implies that the composition $\psi_{t}:=\pi \circ e_{t}$ is an immersion, where $\pi$ is the projection of $X \backslash\left\{x_{0}\right\}$ onto the distance sphere $S_{4 r_{0}}\left(x_{0}\right)$ along the integral curves of $\operatorname{grad} \rho$. Hence $\psi_{t}$ is a covering map and, since the distance sphere is simply connected, a diffeomorphism. It follows that $e_{t}$ is an embedding for $t \geq t_{0}$.

We will prove that $e_{t}$ is an embedding for all $t \geq 0$. Let us assume the contrary and let $\tau:=\sup \left\{t \geq 0 \mid e_{t}\right.$ is not an embedding $\}$. Since the set of all $t$ such that $e_{t}$ is an embedding is open, $e_{\tau}$ is not an embedding. Let $A:=\left\{s \in S \mid e_{\tau}^{-1}\left(e_{\tau}(s)\right) \neq\{s\}\right\}$. Then $A$ is not empty and closed since $e_{\tau}$ is an immersion. We claim: $A$ is open and $e_{\tau}$ is totally geodesic on $A$. It then follows that $A=S$ and $e_{\tau}$ is a totally geodesic immersion of $S$. This is a contradiction since there are no compact totally geodesic submanifolds in $X$.

To prove the claim let $N(s)=\left(d / d t_{\mid t=\tau}\right) e_{t}(s)$ be the normal vectorfield of $e_{\tau}$. Let $p \in A$. Then there exists a point $q \in S$ with $x=e_{\tau}(p)=e_{\tau}(q)$. Since $e_{t}$ is an embedding for all $t>\tau$ it follows $N(p)=-N(q)$. Define $Y=$ $\exp _{x}\left(N^{\perp}(p)\right)$ and let $X_{1}, X_{2}$ be the closure of the two components of $X \backslash Y$ where $N(p)$ points towards $X_{1}$ and $N(q)$ towards $X_{2}$. We consider small neighborhoods $U_{1}, U_{2} \subset S$ of $p$ resp. $q$ and the embedded hypersurfaces $Q_{i}=$ $e_{\tau}\left(U_{i}\right)$. Since these hypersurfaces are convex, it follows that $Q_{1} \subset X_{2}$ and $Q_{2} \subset$ $X_{1}$. If $Q_{1}$ and $Q_{2}$ were not completely contained in $Y$ for sufficiently small $U_{1}, U_{2}$, then $e_{t}\left(U_{1}\right)$ and $e_{t}\left(U_{2}\right)$ intersect for some $t>\tau$ which contradicts to the definition of $\tau$. Thus we can choose $U_{1}$ and $U_{2}$ such that $e_{\tau}\left(U_{1}\right)=$ $e_{\tau}\left(U_{2}\right) \subset Y$. Thus $A$ is open and since $e_{\tau}\left(U_{1}\right) \subset Y$ it follows that $e_{\tau}$ is totally geodesic at $p$ which proves the claim.

For the proof of the rank one case we need a similar result.

Lemma 2. Let $X$ be a Hadamard manifold of dimension $n \geq 3$ with curvature $K \leq-1$ and let $\varphi: S \rightarrow X$ be a compact immersed hypersurface such that the 
second fundamental form with respect to a unit normal vectorfield $N$ along $\varphi$ has eigenvalues $\geq-1$. Then the normal exponential maps $e_{t}: S \rightarrow X, e_{t}(s)=$ $\exp _{\varphi(s)} t \cdot N(s)$ are immersions for all $t \geq 0$ and there exists $a t_{0} \geq 0$ such that $e_{t}$ is an embedding for al $t \geq t_{0}$.

Proof. The curvature assumptions imply that the immersed hypersurface $S$ has no focal points with respect to the normal $N$ and thus $e_{t}$ is an immersion for all $t \geq 0$. The argument of the first part of the proof of Lemma 1 implies that $e_{t}$ is an embedding for $t$ large enough.

Proof of Theorem 1. Let $S:=\partial M$ and define for $\varepsilon>0$ the set $U_{\varepsilon}=$ $\{p \in M \mid \operatorname{dist}(p, S) \leq \varepsilon\}$. For $\varepsilon$ sufficiently small $U_{\varepsilon}$ is contained in $U$ and diffeomorphic to $S \times[-\varepsilon, 0]$ where $(p, t)$ corresponds to $\exp _{p} t \cdot N_{p}$ and $N_{p}$ denotes the outer unit normal. By assumption, $U_{\varepsilon}$ is locally isometric to a symmetric space $X$. Since $S$ is simply connected there exists an isometric immersion $D_{0}: S \times[-\varepsilon, 0] \rightarrow X$ (developing map, see [T]). The assumptions on the curvature imply that the immersed hypersurface has no focal points and we can extend $D_{0}$ to an immersion $D: S \times[-\varepsilon, \infty) \rightarrow X$. The pull back metric on $S \times[-\varepsilon, \infty)$ is locally symmetric and agrees on $S \times[-\varepsilon, 0]$ with the given metric. Thus there exists an isometric embedding $h: M \rightarrow W$ where $W$ is a complete open manifold which is locally isometric to $X$ outside of $h(M)$. Since $W$ is simply connected at infinity one checks easily that $W$ is simply connected and hence a Hadamard manifold.

Note that by Lemma 1 and Lemma $2 D_{\mid S \times\left[t_{0}, \infty\right)}$ is an embedding for $t_{0}$ sufficiently large. Let $\Omega \subset X$ be the complement of the smallest ball containing $D\left(S \times\left\{t_{0}\right\}\right)$ then $f:=\left(D_{\mid \Omega}\right)^{-1}: \Omega \rightarrow W$ is a totally geodesic isometric embedding.

If $\operatorname{rank} X \geq 2$ then by Gromov's rigidity theorem [BGS, $\S 5$ ], $f$ extends to an isometry $f: X \rightarrow W$ and the conclusion follows. If $\operatorname{rank} X=1$, then $f$ extends to an isometry $\bar{f}: X \rightarrow W$ by [SZ, Theorem 7]. (Note that $\partial \Omega$ is convex.)

\section{Proof of Theorem 2}

The proof of Theorem 2 is based on the following

Lemma 3. Let $X^{m+1}$ be a Hadamard manifold and $S \rightarrow X$ a convex embedding of the compact hypersurface $S$. Let $S_{t}$ denote the outer parallel hypersurfaces of distance $t \geq 0$ with total curvature $I(t)=\int_{S_{t}}\left|\operatorname{det} L_{t}\right| d V_{t}$. Then the derivative $I^{\prime}(t)$ is $\geq 0$ for all $t \geq 0$. If $S \rightarrow X$ is strictly convex then equality holds iff for all radial 2-planes $\sigma$ with footpoint lying on $S_{t}$ the curvature $K(\sigma)$ is zero. (By a radial 2-plane we mean a plane which contains the tangent vector of a geodesic starting orthogonally from $S$.) 
Proof of Lemma 3. As in the proof of Lemma 1 we consider the map

$$
\begin{gathered}
e: S \times[0, \infty) \rightarrow X \\
e(s, t):=e_{t}(s):=\exp _{s} t \cdot N(s)
\end{gathered}
$$

where $N$ denotes the outer unit normal field along $S$. Furthermore consider the (1-1)-tensor $\bar{L}_{t}$ defined on the vectorfields on $S$ by $\bar{L}_{t} \stackrel{\text { def }}{=} P_{-t} \circ L_{t} \circ P_{t}$, where $P_{t}$ denotes the parallel transport up to distance $t$ along the geodesics $t \mapsto e(s, t)$ and $L_{t}$ is the Weingarten map of $S_{t}$ with respect to the outer unit normal. Let $d \bar{V}_{t}=f_{t} \cdot d V_{0}$ (with $f_{t} \in C^{\infty}(S)$ ) be the volume element of $S$ induced by the map $e_{t}: S \rightarrow S_{t}$. Then

$$
I(t)=\int_{S} \operatorname{det} \bar{L}_{t} \cdot f_{t} d V_{0}
$$

and

$$
I^{\prime}(t)=\int_{S} \partial\left(\operatorname{det} \bar{L}_{t} \cdot f_{t}\right) d V_{0}
$$

where $\partial:=d / d \tau$. Fix $(s, t) \in[0, \infty)$ and let $e_{1}, \ldots, e_{m}$ be an orthonormal basis of eigenvectors of $\bar{L}_{t}(s)$ with eigenvalues $\lambda_{1}, \ldots, \lambda_{m}$. Note that $\lambda_{i} \geq 0$ for all $i$. Define

$$
\mu_{i}:=\left\langle R\left(P_{t} e_{i}, \dot{\gamma}(t)\right) \dot{\gamma}(t), P_{t} e_{i}\right\rangle \leq 0
$$

where $\gamma(\tau):=e(s, \tau)$. Using the Riccati-equation for $\bar{L}_{t}$ a direct calculation shows:

$$
\begin{aligned}
A: & =\delta\left(\operatorname{det} \bar{L}_{t}(s) \cdot f_{t}(s)\right) \\
& =\left\{-\sum_{i=1}^{m}\left(\lambda_{i}^{2}+\mu_{i}\right) \cdot \prod_{j \neq i} \lambda_{j}+\operatorname{det} \bar{L}_{t}(s) \cdot \operatorname{trace}\left(\bar{L}_{t}(s)\right)\right\} \cdot f_{t}(s) .
\end{aligned}
$$

Observe that $A \geq 0$ if $\operatorname{det} \bar{L}_{t}(s)=\prod \lambda_{i}=0$. If $\operatorname{det} \bar{L}_{t}(s) \neq 0$ then

$$
A=f_{t}(s) \cdot \operatorname{det} \bar{L}_{t}(s) \cdot \sum_{i=1}^{m}-\frac{\mu_{i}}{\lambda_{i}} \geq 0
$$

and equality holds if and only if all $\mu_{i}$ are zero. As $X$ has nonpositive curvature this implies that for all $i$ the vector $P_{t} e_{i}$ is an eigenvector of the endomorphism

$$
x \mapsto R(x, \dot{\gamma}(t)) \dot{\gamma}(t)
$$

for the eigenvalue 0 . Thus $R(\cdot, \dot{\gamma}(t)) \dot{\gamma}(t)=0$.

Proof of Theorem 2. By Lemma $1 S$ is embedded. For small $t_{0}<0$ the inner parallel hypersurface $S_{t_{0}}:=e\left(S, t_{0}\right)$ is still embedded and strictly convex. Thus $S_{t_{0}}$ is diffeomorphic to a sphere and hence the Gauss-Bonnet theorem together with the Gauss equation implies:

$$
I\left(t_{0}\right) \geq 4 \pi
$$

As the curvature is zero for all 2-planes tangent to $S$ the total curvature $I(0)$ is equal to $4 \pi$ and thus $I\left(t_{0}\right) \geq I(0)$. By Lemma 3 this implies that $I(t)=I(0)$ 
for all $t \in\left[t_{0}, 0\right]$ and $K\left(\sigma_{t}\right)=0$ for all radial 2-planes $\sigma_{t}$ with footpoint lying on $S_{t}$. Furthermore $I(t)=4 \pi$ is only possible if all 2-planes tangent to $S_{t}$ have curvature zero. Since $K \leq 0$ this implies that all 2-planes with footpoint on $S_{t}$ have curvature zero.

Let $R$ denote the convex region bounded by $S=\partial R$. Then the above argument shows that $R$ is flat in some neighborhood of the simply connected boundary $S$ and thus Theorem 1 implies that $R$ is flat.

Remark. With the same method one can prove a dual result:

Let $M$ be a compact Riemannian manifold of dimension 3 and with nonnegative sectional curvature. Assume that the boundary $\partial M$ is strictly convex and that $K(\sigma)=0$ for all 2-planes $\sigma$ which are tangent to $\partial M$. Then $M$ is flat.

\section{Proof of Theorem 3}

By Lemma $1 S$ is embedded and we may consider $S$ as a subset of $X$. The monotonicity of $I(t)$ follows from Lemma 3 . We will show that $I(\infty) \leq \omega_{n-1}$ implies that $X$ is isometric to $\mathbf{R}^{n}$ :

If $I(\infty) \leq \omega_{m}$ where $m=n-1$ then $I(t) \leq \omega_{m}$ for all $t \geq 0$. Let $e: S \times[0, \infty) \rightarrow X$ be the normal exponential map as in Lemma 1 and let $L_{t}$ denote the Weingarten map of $S_{t}=e_{t}(S)$ with respect to the outer unit normal. As $S$ is strictly convex there exists a positive number $\mu$ such that all eigenvalues of $L_{0}$ are $\geq 1 / \mu$. Then a comparison argument shows that all eigenvalues of $L_{t}$ are $\geq 1 /(\mu+t)$. Thus

$$
t^{m} \cdot \operatorname{det} L_{t} \geq(1+\mu / t)^{-m} \rightarrow 1 .
$$

Therefore for given $\varepsilon \in(0,1)$ there exists $t_{0} \geq 0$ such that

$$
t^{m} \cdot I(t) \geq(1-\varepsilon) \operatorname{vol}\left(S_{t}\right)
$$

for all $t \geq t_{0}$. We may assume without loss of generality that $t_{0}=0$. Let $B_{t}$ denote the compact region bounded by $S_{t}$. Then

$$
\begin{aligned}
\operatorname{vol}\left(B_{t}-B_{0}\right) & =\int_{0}^{t} \operatorname{vol}\left(S_{\tau}\right) d \tau \\
& \leq(1-\varepsilon)^{-1} \cdot \omega_{m} \cdot \int_{0}^{t} \tau d \tau \\
& \leq(1-\varepsilon)^{-1} \cdot \theta_{n} \cdot t^{n}
\end{aligned}
$$

where $\theta_{n}$ denotes the volume of an Euclidean ball of radius 1. Fix $x_{0} \in B_{0}$. Then $B_{t}\left(x_{0}\right):=\left\{x \in X \mid \operatorname{dist}\left(x_{0}, x\right)<t\right\}$ is clearly contained in $B_{t}$ and thus the above equality implies:

$$
\limsup _{t \rightarrow \infty} \frac{\operatorname{vol}\left(B_{t}\left(x_{0}\right)\right)}{t^{n}} \leq \limsup _{t \rightarrow \infty} \frac{\operatorname{vol}\left(B_{t}\right)}{t^{n}} \leq \theta_{n} .
$$

Since the volume grows as in Euclidean space, $X$ is isometric to $\mathbf{R}^{n}$ [BGS, §1]. 


\section{ACKNOWLEDGMENT}

We would like to thank the referee for his comments.

\section{REFERENCES}

[BGS] W. Ballmann, M. Gromov and V. Schroeder, Manifolds of nonpositive curvature, Birkhäuser, Basel-Boston, 1985.

[GW] R. Greene and H. Wu, Gap theorems for noncompact Riemannian manifolds, Duke Math. J. 49 (1982), 731-756.

[H] J. Hadamard, Sur certaines propriétés des trajectoires en dynamique, J. Math. 3 (1887), 331387.

[S] R. Sacksteder, On hypersurfaces with nonnegative sectional curvature, Amer. J. Math. 82 (1960), 609-630.

[SZ] V. Schroeder and W. Ziller, Local rigidity of symmetric spaces, (preprint), Univ. of Pennsylvania, 1987.

[T] W. Thurston, The geometry and topology of 3-manifolds, Lecture Notes, Princeton University, Princeton, NJ, 1978.

Math. INSTitut der Universität, EInsteinstr. 62, 4400 MÜNSTer, Federal Republic of GERMANY

Department of Mathematics, State University of New York, Stony Brook, New York 